\title{
The Preparation of Congenic Strains of Tetrahymena
}

\author{
SALLY LYMAN ALLEN and PATRICIA H. T. LEE
}

Depts. of Botany and Zoology, Univ. of Michigan, Ann Arbor, Mich. 48104

SYNOPSIS. Congenic strains of syngen 1, Tetrahymena pyriformis, were produced by backcrossing the $\mathrm{F}_{1}$ hybrid between inbred strains $\mathrm{C} 2$ and $\mathrm{D}$ to strain $\mathrm{D}$ in 12 consecutive backcrosses, with selection for certain C2 genes, and then using genomic exclusion to induce homozygosity. Six congenic strains of high breeding performance are available. Five differ from strain $D$ in single genes at 5 different loci. The 6 th strain differs at all 5 loci. Assuming the size of the Drosophila gene ( $4 \times 10^{4}$ nucleotide pairs),

$\mathrm{F}^{\mathrm{O}}$ OR the analysis of biochemical markers control over the residual genotype is a desirable goal. Particularly useful is a set of strains which have identical genotypes except for single gene substitutions. Such congenic strains have been valuable in the refined biochemical characterization of genetic variants in a number of organisms. For example, they have been extremely important in the study of the transplantation antigens in mice (19).

Several genetically determined protein variants occur within inbred strains of syngen 1 of Tetrahymena pyriformis, but these strains differ in their genetic backgrounds. Altho they originated from outcrosses within and between animals taken from only 4 different natural habitats and must share many genes, they have become differentiated from each other during the course of inbreeding (14; see Table 2 in 6 ). To insure strains with similar genetic backgrounds it was therefore necessary to produce congenic strains by appropriate breeding methods.

Congenic strains of high breeding performance are now available. They were produced by repeated backcrosses and selection for certain genes and by making use of genomic exclusion to induce homozygosity. The methods and results of these crosses are documented in this report.

\section{MATERIALS AND METHODS}

Genes in syngen 1.

$m t=$ mating types

$H=$ serotypes expressed at $20-35 \mathrm{C}$

$T=$ serotypes expressed at $38-40 \mathrm{C}$

$E-1=$ esterase-1, observed by starch-gel electrophoresis; substrate: $\alpha$-naphthyl propionate, pH 7.4

$E-2=$ esterase-2, observed by starch-gel electrophoresis; substrate: $\alpha$-naphthyl butyrate, $\mathrm{pH} 6.5$

$P-1=$ phosphatase-1, observed by starch-gel electrophoresis; substrate: sodium $\alpha$-naphthyl acid phosphate, $\mathrm{pH} 5.0$

Linkage relationships. There are 5 pairs of chromosomes in the micronucleus (17). Of the 6 genes listed above only 2 are linked, $m t$ and $E-1$, recombination being $25 \%$ (1). The other genes appear to assort independently or are very loosely linked $(1,6,15$, 16).

Strains. Two inbred strains, D and C2, were used to generate the congenic strains. Strain D was derived from an outcross of

\footnotetext{
'Supported by a Research Grant, GM 15879, from the National Institute of General Medical Sciences, U.S. Public Health Service.
}

we can calculate that strains differing from $D$ by single genes have a heterozygous segment 3 genes long while the strain which differs from $D$ by 5 genes has 5 heterozygous segments and 15 genes contributed from strain C2. A 40-fold increase in heterozygosity would be found with a gene size of $10^{3}$ nucleotide pairs. This means that in using these strains for biochemical work we must be aware that some genetic noise still remains.

an animal taken from a ditch near Alpena, Michigan (ALP-4) to strain B; it has been inbred for 21 generations. Strain C2 was derived by genomic exclusion from a cross of a heterozygote between strains $\mathrm{A}$ and $\mathrm{C}$ and $\mathrm{C}^{*}$, with selection of a strain homozygous for particular genes contributed from the $\mathrm{C}$ strain (which originated from a cross of an animal isolated from a pond in Vermont, UM-226, and strain B) and strain A (which, like strain B, originated from a cross of 2 animals, WH-6 and WH-14, taken from a pond at Woods Hole, Mass.). The genotypes of strains D and $\mathrm{C} 2$ are:

$\mathrm{D} m t^{\mathrm{D}} / m t^{\mathrm{D}} ; H^{\mathrm{D}} / H^{\mathrm{D}} ; T^{\mathrm{C}} / T^{\mathrm{C}} ; E-1^{\mathrm{B}} / E-1^{\mathrm{B}} ; E-2^{\mathrm{B}} / E-1^{\mathrm{B}} ; P-l^{\mathrm{A}} / P-1^{\mathrm{A}}$

$\mathrm{C} 2 m t^{\mathrm{C}} / m t^{\mathrm{C}} ; H^{\mathrm{E}} / H^{\mathrm{E}} ; T^{\mathrm{B}} / T^{\mathrm{B}} ; E-I^{\mathrm{C}} / E-1^{\mathrm{C}} ; E-2^{\mathrm{C}} / E-2^{\mathrm{C}} ; P-1^{\mathrm{B}} / P-1^{\mathrm{B}}$

Culture technics. All crosses and isolations were carried out at $23 \mathrm{C}$. Crosses were made by mixing cells washed in Dryl's salt solution (11). Mating was stopped within $12 \mathrm{hr}$ by the addition of bacterized medium (Cerophyl rye grass inoculated with Aerobacter aerogenes), and individual pairs were isolated into separate containers. A sample of each exconjugant population which developed was tubed up in bacterized medium and tested for its maturity, $H$ and $T$ serotypes, esterase-1, esterase-2, and phosphatase- 1 , the $T$ serotypes and enzymes being tested under axenic conditions.

Tests for maturity were carried out by addition of testers of either mating type IV or VII to samples of the exconjugant cultures. The absence of mating pairs shows that the exconjugant culture is "immature" and has undergone normal conjugation.

For the cross employing genomic exclusion, exconjugant cultures are mature and remate, so a 2 nd set of isolations is necessary (3). Tests for maturity, serotypes and isozymes were carried out on samples of the Round 2 exconjugants.

In syngen 1 maturity sets in after 60-80 fissions. In order to produce sexually reactive progeny which could be used in subsequent crosses it was therefore necessary to initiate clones from each exconjugant culture and to transfer them serially by single cell isolations at 13 fission intervals. Mating type tests were then carried out on the mature lines.

Identification of serotypes. The $\mathrm{H}$ serotypes were scored by mixing samples of bacterized cells grown at $23 \mathrm{C}$ with each type of antiserum (anti-Hd and anti-He). Immobilization of cells is specific and indicates the presence of a particular antigen. Homozygotes have only one of the 2 antigens while heterozygotes (at the time tested) have both antigens.

The $\mathrm{T}$ serotypes were tested in cells grown in liver-peptone $(0.4 \%$ liver fraction $\mathrm{L}, 1 \%$ proteose-peptone) at $38.5 \mathrm{C}$. Samples of cells, rendered bacteria free by passage thru antibiotics and grown at $30 \mathrm{C}$ in $1 \%$ proteose-peptone, were subcultured into liver-peptone $(0.7 \mathrm{ml}$ into $5 \mathrm{ml})$ and grown for exactly $25 \mathrm{hr}$ at $38.5 \mathrm{C}$. After $1 \mathrm{hr}$ at $23 \mathrm{C}$, samples of cells were mixed with each type of antiserum (anti-Tb and anti-Tc). Homozygotes are immobilized by only one of the antisera while heterozygotes are immobilized by both antisera. 
TABLE 1. Viability of backcrosses.

\begin{tabular}{|c|c|c|c|c|}
\hline Generatio & Parents & $\begin{array}{c}\text { Total } \\
\text { Isolated }\end{array}$ & $\begin{array}{c}\text { No. } \\
\text { Immature }\end{array}$ & $\begin{array}{c}\% \\
\text { Viable }\end{array}$ \\
\hline$F_{1}$ & $\mathrm{C} 2-3686 \mathrm{c} \times \mathrm{D}-1968 / \mathrm{b}$ & 30 & 30 & 100 \\
\hline $\mathrm{BC}_{1}$ & $520-6 a($ II $) \times$ D-19683b & 180 & 165 & 92 \\
\hline $\mathrm{BC}_{2}$ & $2-153 a(V I) \times D-19683 b$ & 270 & 253 & 94 \\
\hline $\mathrm{BC}_{3}$ & $8-29 c(V I) \times D-19683 b$ & 270 & 233 & 87 \\
\hline $\mathrm{BC}_{4}$ & $12-67 \mathrm{c}(\mathrm{I}) \times \mathrm{D}-19686$ & 270 & 250 & 93 \\
\hline $\mathrm{BC}_{5}$ & $32-86 a($ III $) \times$ D-20692 & 270 & 266 & 99 \\
\hline $\mathrm{BC}_{\mathbf{s}}$ & $59-19 b(\mathrm{I}) \times \mathrm{D}-20693$ & 270 & 250 & 93 \\
\hline $\mathbf{B C}_{7}$ & $68-51 \mathbf{a}(\mathrm{V}) \times \mathrm{D}-20692$ & 270 & 245 & 90 \\
\hline $\mathrm{BC}_{8}$ & $76-6 a(I I) \times D-20693$ & 270 & 238 & 92 \\
\hline $\mathrm{BC}_{0}$ & $92-12 c(V I) \times D-20693$ & 270 & 259 & 96 \\
\hline $\begin{array}{ll}\mathbf{B C}_{10} & 1\end{array}$ & $114-203 b(V) \times D-20693$ & 270 & 256 & 95 \\
\hline $\mathrm{BC}_{\mathbf{1 1}}$ & $126-58 \mathrm{a}(\mathrm{II}) \times \mathrm{D}-20693$ & 270 & 249 & 92 \\
\hline $\mathrm{BC}_{12}$ & $131-82 a(I) \times D-20693$ & 270 & 260 & 96 \\
\hline
\end{tabular}

Identification of esterase and phosphatase isozymes. $100 \mathrm{ml}-$ samples of cells grown at $30 \mathrm{C}$ for 1 week in $1 \%$ proteose-peptone were concentrated by centrifugation. Frozen-thawed extracts of concentrated cells were then subjected to starch-gel electrophoresis, and the esterases and phosphatases visualized in the gels by incubation in appropriate reaction mixtures $(2,7)$. The gels were scored by visual examination and photographed.

\section{RESULTS}

Backcrosses. An $F_{1}$ heterozygote was produced from a cross of inbred strains $\mathrm{C} 2$ and $\mathrm{D}$ and then backcrossed to strain D. A total of 12 backcrosses to strain D was carried out. In each backcross generation, except for the first, 270 pairs were isolated and all the immature progeny were tested for their $\mathrm{H}$ serotypes. The Hde heterozygotes were then selected, tested for their $\mathrm{T}$ serotypes, and the Tbc heterozygotes selected and tested for their esterases. The E-1bc E-2bc heterozygotes were then tested for their phosphatases. From each generation an average of 4 progeny which were heterozygous for the genes $H, T$, $E-1, E-2$, and $P-1$ was produced. No attempt was made to select for heterozygotes for the $m t$ gene since both strains C2 and D give similar arrays of mating types ( $I$, II, III, V, and VI) altho in different frequencies.

From each of the progeny mature lines were obtained by serial transfer of clones, tested for mating type, and crossed to strain D. The cross with the highest breeding performance was then selected for the next backcross generation.

As shown in Table 1, the breeding performances of the backcross generations were excellent. Viabilities (\% immature progeny) ranged from 87 to $99 \%$, the average for all 12 generations being 94\%. To insure high breeding performance of the $\mathrm{D}$ strain parent during the course of the backcrosses, strain D was inbred 3 times during the year that it took to complete the backcrosses.

With an average of $94 \%$ viability, 254/270 immature progeny would be expected. Since we would expect a
TABLE 2. Backcross Segregation Data.

\begin{tabular}{lccl}
\hline & $(\mathrm{C} 2 \times \mathrm{D}) \mathrm{D}:$ & \multicolumn{2}{c}{$(7 \times 8) 7:$} \\
\hline Hde/Total & $1431 / 2924$ & $741 / 1540 \quad(\mathrm{Hae})$ \\
Tbc & $702 / 1431$ & $335 / 730^{*}$ & $(\mathrm{Tab})$ \\
E-1bc & $388 / 678$ & $184 / 335$ & \\
E-2bc & $100 / 388$ & $77 / 184$ & \\
P-1ab & $50 / 100$ & $40 / 77$ & \\
\hline
\end{tabular}

* Deficiency of Tab heterozygotes resulted from the technical difficulty of distinguishing Tab from the Ta homozygote.

1:1 segregation of heterozygotes and homozygotes for each gene, out of 254 we would predict 8 , or $1 / 32$, of the progeny to be heterozygous for all 5 genes. An average of only 4 such progeny was observed. This deficiency could be explained if an additional gene difference were involved.

Segregation data for all the backcross progeny are summarized in Table 2. For each gene a 1:1 segregation of heterozygotes and homozygotes is expected. No significant departure from this ratio was observed for genes $H, T$ and $P-1$. A significant excess of heterozygotes was observed for $E-1$, and a highly significant deficiency of heterozygotes was observed for $E-2$ (chi-square tests gave $\mathrm{p}$ values of $<0.0001)$. Similar trends were also apparent in another series of backcrosses involving strains 7 and 8, shown in the 2nd column of Table 2. In the case of E-1, weak linkage to either $H$ or $T$ might explain the excess of heterozygotes. However, previous studies have not provided evidence that $E-1$ is linked to either of these loci, altho it is linked to the $m t$ locus $(1,6,16)$. In the case of $E-2$ the observed heterozygotes were about half the number expected in the $(\mathrm{C} 2 \times \mathrm{D}) \mathrm{D}$ backcross series. About $1 / 4$ of the gels showed little or no activity for $E-2$, and there were gradations in activity where activity was seen. It is therefore possible that there is a gene contributed from strain $\mathrm{C} 2$ which is necessary for activity of the product of the $E-2^{c}$ allele. Genes affecting the activity of enzymes have not been detected previously in Tetrahymena but they have been found in certain stocks of Paramecium aurelia and affect particular classes of esterases (10).

Induction of homozygosity by genomic exclusion. Autogamy, or the self-fertilization of a single animal, results in homozygosity of all genes. This occurs in some groups of ciliated protozoa like $P$. aurelia but it does not occur in $T$. pyriformis. However, there are certain abnormal strains of Tetrahymena which, when crossed to normal strains, do not contribute any of their genes to the progeny but cause the genes present in the normal strain to become homozygous in the progeny. This abnormal form of conjugation is called genomic exclusion and it has the same net effect as autogamy. A strain called $\mathrm{C}^{*}$ always undergoes genomic exclusion when crossed to normal strains. This strain was then used in a cross to the 12th backcross generation to produce homozygotes. 
TABLE 3. Viability of $B C_{u} \times C^{*}$.

\begin{tabular}{|c|c|c|c|c|c|c|}
\hline \multicolumn{2}{|c|}{ Parents } & \multicolumn{2}{|c|}{ Round 1} & \multicolumn{3}{|c|}{ Round 2 Sets } \\
\hline $\mathrm{BC}_{12}$ & $\mathrm{C}^{*}$ & $\begin{array}{c}\text { Total } \\
\text { Isolated }\end{array}$ & $\begin{array}{c}\% \\
\text { Mature }\end{array}$ & $\begin{array}{c}\text { Total } \\
\text { Isolated }\end{array}$ & $\begin{array}{c}\text { No. } \\
\text { Immature }\end{array}$ & $\begin{array}{c}\% \\
\text { Viable }\end{array}$ \\
\hline \multicolumn{2}{|c|}{$141-269 c(\mathrm{VI}) \times 45-6-39$} & 600 & 92 & 68 & 64 & 94 \\
\hline \multicolumn{2}{|c|}{$141-269 c($ VI $) \times 45-10-16$} & 6600 & 96 & 85 & 77 & 91 \\
\hline \multicolumn{2}{|c|}{$141-62 a($ II $) \times 45-10-16$} & 600 & 95 & 172 & 157 & 91 \\
\hline
\end{tabular}

Before making the cross $\mathrm{BC}_{12} \times \mathrm{C}^{*}$, the $\mathrm{C}^{*}$ parent was cloned since structurally abnormal animals with gross defects in the micronucleus are present in the population. Twenty clones which contained structurally normal animals were established by rigorous selection and then pretested in crosses to the $\mathrm{D}$ strain parent. Three clones which showed $97-100 \%$ mature progeny in these crosses were then selected and crossed on a small scale to 5 different $\mathrm{BC}_{12}$ lines. The 3 best crosses were then repeated on a large scale.

Where genomic exclusion occurs in a cross, 2 rounds of mating occur. Exconjugants from Round 1 are heterocaryons, having an old macronucleus and a homozygous new micronucleus; when they come apart after conjugation, each exconjugant replicates and, when the food is exhausted some 2-3 days later, the 2 populations remate and give rise to a population of genetically identical mating pairs $(3,4)$. These are the Round 2 exconjugants; they are immature and also homozygous. Genotypically different homozygous strains result from different acts of genomic exclusion. Any desired genotype can be selected.

In practise what we did was to isolate individually 600 Round 1 pairs from 3 different $\mathrm{BC}_{12} \times \mathrm{C}^{*}$ crosses. Beginning 2 days later the different containers were examined and, whenever we saw pairs, we isolated 6 of these Round 2 pairs into separate containers. After 3 more days the 2nd set of cultures was examined for death and maturity. A sample of the cultures which were alive and which did not contain pairs was then tubed up. To the left-over sample in the containers, tester VII was added and the containers were examined for pairs the following day. Only immature cultures were used for testing phenotypes.

The vital statistics of the $\mathrm{BC}_{12} \times \mathrm{C}^{*}$ crosses are summarized in Table 3. The viabilities of both the Round 1 and Round 2 exconjugants were high in all 3 crosses. Among the Round 2 sets there was considerable uniformity in response: all 6 replicate cultures were either dead, mature or immature. We ended up with a total of 298 sets of Round 2 progeny which we could assay for phenotype.

TABLE 4. $B C_{1} \times C^{*}$ segregation data.

\begin{tabular}{lccccc}
\hline \hline $\begin{array}{l}\text { Source } \\
\text { of Allele }\end{array}$ & $H$ & $T$ & $E-1$ & $E-2$ & $P-1$ \\
\hline Strain D & d 166 & c 159 & b 162 & b 161 & a 51 \\
Strain C2 & e 132 & b 139 & c 136 & c 137 & b 42 \\
\hline
\end{tabular}
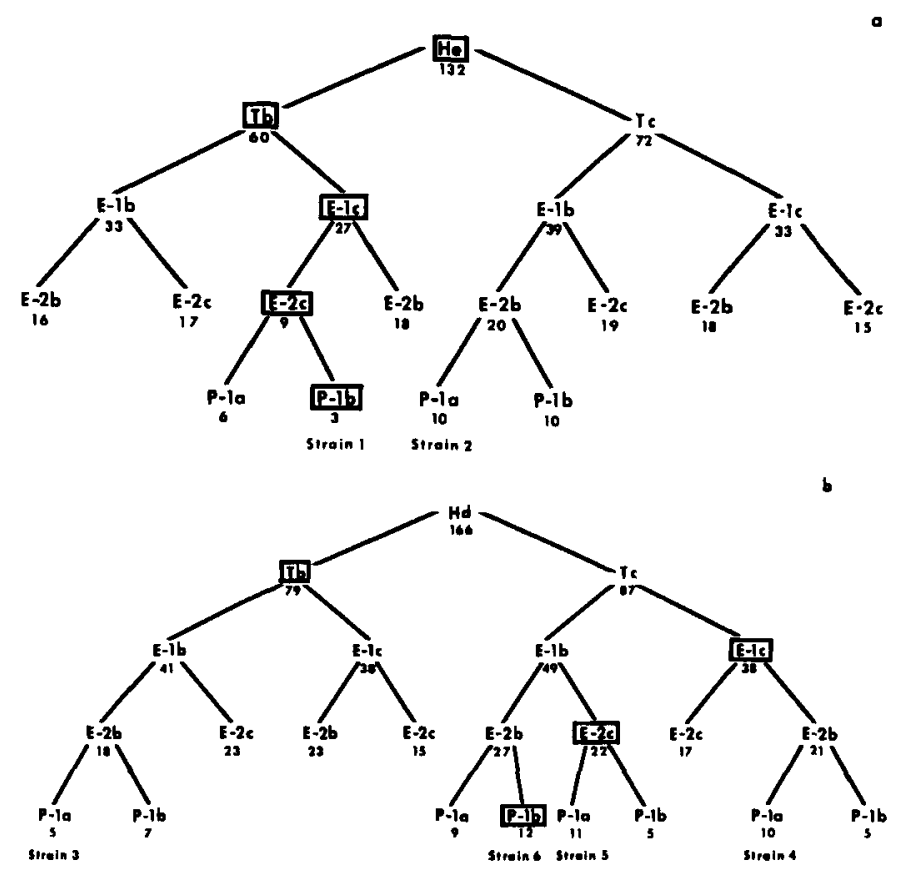

Fig. 1. Detailed segregation data from $B_{12} \times C^{*}$, (a) Congenic strains 1 and 2, (b) Congenic strains $3,4,5$, and 6. C2 phenotypes which were selected are enclosed in boxes.

We selected the first culture of each of the 298 sets for testing and examined them for their serotypes and isozymes. Then from the progeny which we were interested in keeping, we typed the remaining replicates of the set. There was complete agreement of the phenotypes within a set.

The results of testing the first cultures of each of the 298 sets of progeny are shown in Table 4 . The data are complete for the first 4 genes, but only selected strains were tested for $P-1$. We would expect a $1: 1$ segregation of strains homozygous for alleles at each of the 5 loci. Curiously, we observed that there was a slight departure in each of the ratios favoring the allele contributed from strain D (average 54\%). None of the individual ratios is significantly different from $1: 1$. Nor, if we partition the data into different classes, is there any significant trend within groups of particular genotypes (Fig. 1). However, if we compare the total number of alleles contributed from the D strain (699) with the total number contributed from the C2 strain (586), there is a significant excess of $D$ genes (chi-square test gives a $p$ value of $<0.01)$. Perhaps D genes confer greater viability to an animal possessing them. The average viability of the Round 2 progeny was 92\% (298/325); 27 progeny either died or were mature. Perhaps all of them had C2 genes. If 27 is added to the observed numbers (except for $P-1$ ), this brings the ratio of alleles very close to $1: 1$.

The congenic strains selected. Six strains congenic with strain D were selected (Table 5). Congenic strains 2-6 have only single genes derived from C2. Strain 1 has 5 genes derived from C2. The number of progeny of each of these genotypes can be found by examining Fig. 1. 
TABLE 5. Genotypes of parental and congenic strains.

\begin{tabular}{|c|c|c|c|c|c|}
\hline Strain & $H$ & $T$ & $E-1$ & $E-2$ & $P-1$ \\
\hline $\mathrm{C} 2$ & E & B & $\mathrm{C}$ & $\mathrm{C}$ & B \\
\hline D & $\mathrm{D}$ & C & B & B & $A$ \\
\hline Congenic-1 & $\underline{E}^{*}$ & B & $\underline{C}$ & C & $B$ \\
\hline 2 & $\underline{E}$ & C & B & L & A \\
\hline 3 & $\mathrm{D}$ & B & B & B & $\mathrm{A}$ \\
\hline 4 & D & C & $\underline{\mathrm{C}}$ & B & $A$ \\
\hline 5 & $D$ & $\mathrm{C}$ & B & $\underline{\mathrm{C}}$ & $A$ \\
\hline 6 & D & C & B & B & B \\
\hline
\end{tabular}

* Alleles derived from C2 strain are underlined.

It varied from 3 (for strain 1) to 11 (for strain 5). In many instances not all possible progeny were selected for testing for $P-1$.

The mating type distributions of the congenic strains are shown in Table 6 . The distributions within the 6 strains are remarkably uniform. This is a significant observation since Nanney (14) noted that differing genetic backgrounds affect mating type frequencies. We may then infer from the uniformity in mating type distributions that the congenic strains have similar genetic backgrounds. If we compare the distributions of the congenic strains to those characteristic of strains C2 and D, we can see that they resemble those found in strain $\mathrm{D}$ and differ significantly from those of strain C2. This means that the $m t^{\mathrm{D}}$ allele rather than the $m t^{\mathrm{C}}$ allele is present in the congenic strains. After 12 backcrosses we would not expect to see the $m t^{\mathrm{C}}$ allele without selection for it.

TABLE 6. Distribution of mating types in congenic and parental strains.

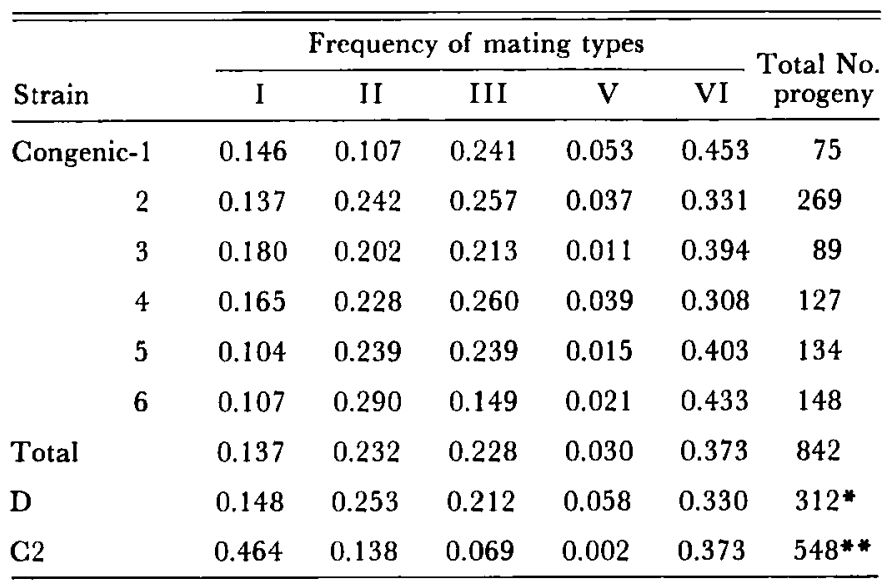

- Data from Nanney (11).

* Unpublished data (S. L. Allen and E. M. Simon).

Chi-square tests:

For homogeneity of congenic strains : $\chi^{2}=23.6$, d.f. $=24, p=0.5$.

Total, congenic strains vs. $D$ strain: $\chi^{2}=6.4$, d.f. $=1, p=0.1-0.2$

Total, congenic strains vs. C2 strain : $\chi^{2}=222$, d.f. $=1, \mathrm{p}<0.0001$.
Selection for $E-1^{\mathrm{c}}$, which is linked to $m t^{\mathrm{c}}$, was carried out. However, this is not a tight linkage and it would appear that this linkage has been broken during the backcrosses and that the $m t^{\mathrm{c}}$ allele has been lost.

Each of the new strains has been tested for its breeding performance by inbreeding. There is some variability in performance of crosses of clones within different lineages, the overall average for all strains being around $80 \%$. However, there are many crosses which give 93$100 \%$ viability. Thus, the prognosis for future work with these strains looks bright.

\section{DISCUSSION AND CONGLUSIONS}

The goal sought in producing congenic strains is that they have similar genotypes except for those gene differences which have been selected during the backcrosses. Has this goal been met? What is the degree of residual heterozygosity that remains after 12 backcrosses? And in terms of numbers of genes, how many gene differences are there between congenic strains?

To start, let us make 2 assumptions. First, we will assume that the 2 inbred strains, C2 and D, differ from each other in all their genes. Second, we will for the moment disregard the effect of linkage. A cross is made and the $F_{1}$ hybrid is backcrossed to strain $D$ in 12 successive backcrosses. The degree of residual heterozygosity is therefore

$$
\frac{1}{2^{12}}=\frac{1}{4096}=0.000244 \text {. }
$$

What does this mean in terms of gene numbers? We can use the amount of DNA in the haploid genome to get at the total number of genes assuming a similar size for each gene. Amounts of DNA have been measured by cytospectrophotometry of the micronucleus and also by determining the rate of renaturation of denatured DNA and relating the rate to the haploid genome size $(5,12,20)$. By these methods the amount of DNA in the haploid genome can be estimated as 0.4-1.6 $\times 10^{11}$ daltons. If we use the value $1.3 \times 10^{11}$ daltons, this is $2 \times 10^{8}$ in terms of nucleotide pairs. This value is similar to that calculated for the Drosophila genome (18).

The number of genes we estimate depends upon the value used for gene size. In Drosophila each band of a polytene chromosome is believed to be equivalent to a single gene and the haploid amount of DNA it contains is estimated at $4 \times 10^{+}$nucleotide pairs (9). There are about 5000 bands and thus 5000 genes in Drosophila. If, however, each gene contained $10^{3}$ nucleotide pairs, then the number of genes would be increased 40 -fold.

If we use the figure 5000 obtained from Drosophila for the number of genes in Tetrahymena, we can then estimate the number of genes heterozygous after 12 backcrosses. Multiplying $5000 \times 0.000244$ gives a value of 1.22. This means that in the 12 th backcross generation there is present on the average only one allele contributed from the C2 strain. If we use the figure $2 \times 10^{5}$ for the 
number of genes, this leads to an estimate of 50 alleles contributed from the $\mathrm{C} 2$ strain.

The genic contribution made by the $\mathrm{C} 2$ strain is further reduced in the cross in which homozygotes are produced by genomic exclusion. Approximately half the remaining heterozygous genes will become homozygous for the C2 alleles, the other half becoming homozygous for the $D$ alleles. This means that on the average $0.5-25$ of the genes present in the congenic strains should be derived from the C2 strain, depending upon the value used for the size of the gene.

We made 2 assumptions in the beginning. First, we assumed that strains $\mathrm{C} 2$ and $\mathrm{D}$ differed completely in genotype. When the inbreeding coefficient is calculated we find that at a minimum they share $1 / 16$ of their genes. This means that the number of gene differences is reduced by the factor $1-1 / 16$, and the residual heterozygosity after 12 backcrosses becomes

$$
\frac{1}{4370}=0.000229 \text {. }
$$

The 2nd assumption we made specifically ignored the effect of linkage. The genome is divided up into 5 metacentric chromosomes, rather similar in length. From the data on $m t$ and $E-1$ we do know that linkages of the order of $25 \%$ are broken, but we don't know how effective our breeding program was in breaking up very tight linkages. No measures of intrachromosomal recombination rate are available for the ciliated protozoa so we cannot assess the degree to which linkage would increase residual heterozygosity. The best we can do is to use a value obtained in other organisms and to assume a cytogenetic map comparable in length to that of Drosophila. Bartlett and Haldane (8) gave the value $\pm 1 / \mathrm{t}$ centimorgans for the length of the heterozygous segment, where $t=$ the number of backcrosses. In our crosses $1 / t=1 / 12$ and the total length of each heterozygous segment would be $1 / 6 \mathrm{cM}$. The total map length in Drosophila is $287.2 \mathrm{cM}$ (13). With 5000 genes, each centimorgan would encompass 18 genes, and $1 / 6 \mathrm{cM}=3$ genes. In congenic strains selected for $1 \mathrm{C} 2$ gene, this means that each of these strains has a heterozygous segment 3 genes in length. Congenic strain 1 would have 5 heterozygous segments on different chromosomes (or on different arms of the chromosomes) and thus a total contribution of 15 genes from C2. If the smaller size of the gene is used, then these values would be raised to 120 and 600 genes, respectively.

The true value probably lies somewhere between. This means that in using these strains for biochemical work we must be aware that some genetic noise still remains.
How much noise depends on the size of the gene and specific factors affecting intrachromosomal recombination in Tetrahymena. Nevertheless, we will be in a much better position to analyze specific protein and nucleic acid differences using pairs of congenic strains.

We would like to thank James $H$. Asher (Univ. of Michigan) for his help in computing the inbreeding coefficient of our parental strains and the contribution to residual heterozygosity of linkage, and Helen Gay (Univ. of Michigan) for advice on the data borrowed from the Drosophila genome.

\section{REFERENCES}

1. Allen, S. L. 1964. Linkage studies in variety 1 of Tetrahymena pyriformis: A first case of linkage in the ciliated protozoa. Genetics 49, 617-27.

2. 1964. The esterase isozymes of Tetrahymena: Their distribution in isolated cellular components and their behavior during the growth cycle. J. Exp. Zool. 155, 349-70.

3. 1967. Genomic exclusion: A rapid means for inducing homozygous diploid lines in Tetrahymena pyriformis, syngen 1 , Science 155, 575-7.

4. 1967. Cytogenetics of genomic exclusion in Tetrahymena. Genetics 55, 797-822.

5. Allen, S. L. \& Gibson, I. 1971. Genome amplification and gene expression in the ciliate macronucleus. Submitted to Nature.

6. 1971. Genetics of Tetrahymena, in Elliott, A. M., ed. The Bialogy of Tetrahymena, Appleton, Century, Crofts, New York (in press)

7. Allen, S. L., Misch, M. S. \& Morrison, B. M. 1963. Variations in the electrophoretically separated acid phosphatases of Tetrahymena. J. Histochem. Cytochem. 11, 706-19.

8. Bartlett, M. S. \& Haldane, J. B. S. 1935 . The theory of inbreeding with forced heterozygosis. J. Genet. 31, 327-40.

9. Berendes, H. D. 1970. Polytene chromosome structure at the submicroscopic level. I. A map of region X, 1-4E of Drosophila melanogaster. Chromosoma 99, 118-30.

10. Cavill, A. \& Gibson, I. 1971. Genetic determination of esterases in Paramecium. To be submitted to Biochem. Genetics.

11. Dryl, S. 1959. Antigenic transformation in Paramecium aurelia after homologous antiserum treatment during autogamy and conjugation. (Abstr.) J. Protozool. 6 (Suppl.), 25.

12. Flavell, R. A. \& Jones, I. G. 1970. Kinetic complexity of Tetrahymena pyriformis nuclear deoxyribonucleic acid. Biochem. J. 116, 155-7.

13. Lindsley, D. L. \& Grell, E. H. 1968. Genetic variations of Drosophila melanogaster. Carnegie Inst. Wash. Pub. No. 627, $471 \mathrm{pp}$.

14. Nanney, D. L. 1959. Genetic factors affecting mating type frequencies in Variety 1 of Tetrahymena pyriformis. Genetics 44, $1173-84$.

15. 1960. The relationship between the mating type and $\mathrm{H}$ serotype systems in Tetrahymena. Genetics 45, 1351-8.

16. Phillips, R. B. 1967. Inheritance of $T$ serotypes in Tetrahymena. Genetics 56, 667-81.

17. Ray, C. Jr. 1956. Meiosis and nuclear behavior in Tetrahymena pyriformis. J. Protozool. 3, 88-96.

18. Ritossa, F. M. \& Spiegelman, S. 1965. Localization of DNA complementary to ribosomal RNA in the nucleolus organizer region of Drosophila melanogaster. Proc. Nat. Acad. Sci., U.S., 53, 737-45.

19. Snell, G. D. \& Stimfling, J. H. 1966. Genetics of tissue transplantation, in Green, E., ed. Biology of the Laboratory Mouse, McGraw-Hill, New York, 457-91.

20. Woodard, J., Gorovsky, M. \& Kaneshiro, E. 1968. Macronuclear subnuclei in Tetrahymena. J. Cell Biol. 39, 192a. 